\title{
Ultrafast dephasing of light in strongly scattering GaP nanowires.
}

\author{
Martina Abb, ${ }^{1}$ Erik P. A. M. Bakkers, ${ }^{2}$ and Otto L. Muskens ${ }^{1}$ \\ ${ }^{1}$ SEPnet and the Department of Physics and Astronomy, \\ University of Southampton, Highfield, Southampton SO17, 1BJ, United Kingdom 常 \\ ${ }^{2}$ Eindhoven University of Technology, P.O. Box 513, 5600 MB Eindhoven, The Netherlands
}

(Dated: February 17, 2011)

\begin{abstract}
We demonstrate ultrafast dephasing in the random transport of light through a layer consisting of strongly scattering GaP nanowires. Dephasing results in a nonlinear intensity modulation of individual pseudomodes which is 100 times larger than that of bulk GaP. Different contributions to the nonlinear response are separated using total transmission, white-light frequency correlation, and statistical pseudomode analysis. A dephasing time of $1.2 \pm 0.2 \mathrm{ps}$ is found. Quantitative agreement is obtained with numerical model calculations which include photoinduced absorption and deformation of individual scatterers. Nonlinear dephasing of photonic eigenmodes opens up avenues for ultrafast control of random lasers, nanophotonic switches, and photon localization.
\end{abstract}

PACS numbers: 42.25Bs, 78.47J-, 78.67Uh

The appearance of an interference pattern after transport of coherent light through a multiple scattering medium is the result of coherent summation of thousands of light paths with random phases. In recent years, new methods have been developed to exploit the coherent aspects of diffuse light transport for imaging [1, 2]. Classical waves, like light or sound, provide unique opportunities to study mesoscopic wave transport, such as localization, in the absence of many-body interactions usually found for electrons [3, 4]. Coherent light scattering has recently lead to exciting new directions of research, such as coupling of localized states with quantum emitters [5], transverse localization, and combined effects of localization and nonlinearity [ $[6]$.

Although the lack of many-body interactions makes phase coherence generally more robust for optical waves than for electrons, several dephasing processes can influence the transport of light, such as magnetic fields, scattering from moving particles, and polarization effects [7 9 ]. The time required for electrons or photons to loose their coherence due to inelastic collisions is known in the theory of electronic conductance as the phase breaking time [10]. While for electrons phase breaking (decoherence) processes have only been measured indirectly through changes in the conductivity with temperature, dephasing can be observed directly for optical waves by the changes in speckle pattern [11], which is used in diffusing wave spectroscopy [12].

In this Letter, we demonstrate a new regime of reversible dephasing in a random medium on ultrafast time scales. The ultrafast dephasing takes place a million times faster than other dephasing mechanisms such as Brownian movement, opening up new applications in ultrafast control of random lasers, quantum emitters, and localization phenomena. The material under study consists of a layer of semiconductor nanowires, which are important for novel applications in optoelectronics and photovoltaics [13 15]. We have recently demonstrated that these semiconductor nanowires form one of the most efficient light trapping layers available to date 16. Here, we combine these favorable scattering properties with the intrinsic nonlinearity of the semiconductor host material to achieve active control on ultrafast time scales.

Gallium phosphide $(\mathrm{GaP})$ nanowires were grown using metallo-organic vapour phase epitaxy as described in Ref. [16]. The length of the nanowires was controlled by the VLS growth time, while the thickness and volume fraction was independently tuned by switching to a lateral growth regime where material was deposited homogeneously over the nanowire sidewalls. In the experiments of this work, we make use of two nanowire samples, respectively with mean free path $\ell=0.16 \pm 0.02 \mu \mathrm{m}$ and thickness $L=1.5 \mu \mathrm{m}$ (sample 10 of Ref. [16], here sample 1), and $L=4.0 \mu \mathrm{m}, \ell=0.2 \pm 0.02 \mu \mathrm{m}$ (sample 11 of Ref. 16], here sample 2), at a wavelength of $632 \mathrm{~nm}$. The variation of $\ell$ with wavelength was found to be small over the spectral range of our experiments [16].

We investigated the photoinduced nonlinear response using a broadband white-light supercontinuum probe generated by focusing of $800-\mathrm{nm}$ laser pulses from a regenerative amplifier (Coherent RegA, $250 \mathrm{kHz}, 200 \pm 10 \mathrm{fs}$ pulse duration) in a quartz window. The material is pumped using the second harmonic at $400 \mathrm{~nm}$ wavelength. Both the pump and probe are focused onto the sample using a lens with a 6 -cm focal length, resulting in a focus of around $5 \mu \mathrm{m}$ in diameter and a pump fluence of $8 \mathrm{~mJ} / \mathrm{cm}^{2}$. Total transmission measurements are shown in Fig. 1(a) for the nanowires as well as for a GaP substrate (thin line, blue).

In absence of absorption, the transmission through a scattering layer follows Ohm's law $T \simeq \ell / L$. The total transmission can be derived including effects of internal reflection [17], which for small absorption $\left(L_{a}>L>\ell\right)$ reads

$$
T=\frac{\ell+z_{e 1}}{\left(1+z_{e 1} z_{e 2} / L_{a}^{2}\right) L+\left(z_{e 1}+z_{e 2}\right)},
$$



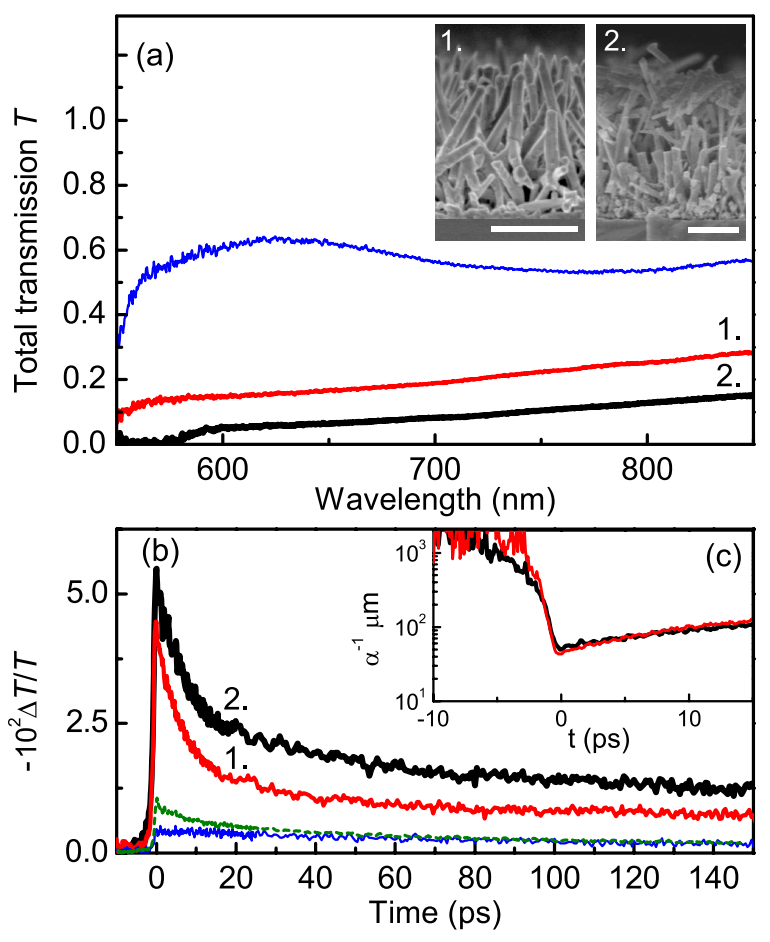

FIG. 1: (color online) (a) Total transmission spectra for nanowire layers 1 and 2, and for a GaP substrate (thin line, blue). Inset: scanning electron microscopy images, scale bars $1 \mu \mathrm{m}$. (b) Pump-probe total transmission $\Delta T / T$ for the samples of (a) and for a 51-nm diameter nanowire layer (dash, sample 6 of Ref. [16]). (c) Absorption length $\alpha^{-1}$ obtained from (b) using Eq. (1).

where $L$ denotes the slab thickness, $z_{e, i}$ are extrapolation lengths which represent the effects of internal reflection at the front and rear interfaces of the diffuse slab. The diffuse absorption length is defined as $L_{a}=(\ell / 3 \alpha)^{1 / 2}$, with $\alpha^{-1}$ the bulk absorption length.

Pump-probe total transmission measurements are shown in Fig. 1(b). All samples show a reduction of the total transmission directly following excitation by the pump laser. Independent experiments were performed verifying that mesoscopic contributions to the total transmission, i.e. fluctuations caused by a finite number of transmission channels [18], were unimportant under the present experimental conditions. Therefore the signals in Fig. 1(b) are attributed to changes in the absorption coefficient of the materials. Transient absorption in semiconductor nanostructures results from free carrier generation [19]. A faster relaxation time is observed for nanowires compared to the bulk semiconductor, which can be attributed to trapping of photogenerated carriers at surface states [20]. The transient relative absorption increases from $3 \times 10^{-3}$ for the GaP substrate to $5.2 \times 10^{-2}$ for nanowire sample 2 . We attribute this strong increase in absorption by light trapping in the nanowire layer 21]. Light trapping can be assessed from the time the light resides in the micrometer-thin photoexcited region, where it is susceptible to nonlinear absorption. For the GaP substrate, the coherent beam traverses this region in around $10 \mathrm{fs}$, while, for nanowire sample 2, the light diffusion time amounts to approximately $L^{2} / \pi^{2} D \simeq 0.15$ ps. We note that optically thin nanowires [dashed line, green in Fig. 1(b)] did not show such a marked increase of the nonlinear absorption, eliminating other possible nanowire surface effects.

Equation 1 can be inverted to yield values for the absorption length $\alpha^{-1}$ for a pump-probe signal $\Delta T / T$, as shown in Fig. 1(c). A minimum absorption length $\alpha^{-1}$ of $49.9 \pm 4 \mu \mathrm{m}$ is found, corresponding to a change of the imaginary refractive index $\Delta \kappa=c_{0} \alpha / 4 \pi \nu$ of $(1.0 \pm 0.2) \times 10^{-3}$. Using a free-carrier absorption cross section of GaP of $1.1 \times 10^{-18} \mathrm{~cm}^{-2}$ [22], our value for $\Delta \kappa$ amounts to a free-carrier density of $2 \times 10^{20} \mathrm{~cm}^{-3}$, which is a realistic density for the strong optical pumping regime under study.

In the following we focus on the results for nanowire sample 2. Similar results are obtained for sample 1 [23]. To assess the effects of nonlinear pumping on the dynamical transport parameters of the random medium, we measured the frequency correlations of light transmitted through the nanowire slab. For this purpose, a cone of light of around $2^{\circ}$ angular width was collected and analyzed using a fiber spectrometer. Broadband linear polarization filters were used both for the incident and for the detected light. Spectra were measured as a function of pump-probe delay time, yielding time-resolved spectral maps such as shown in Fig. 2(a). The transmission spectra consist of large fluctuations corresponding to frequency speckle. Within 1 ps following excitation with a pump laser, changes occur in both the spectral position and amplitude of the fluctuations, as is illustrated in Fig. 2(b) where we compare two spectra taken respectively 3 ps before and 1 ps after arrival of the pump pulse. To quantify these variations in the speckle pattern, we calculated the time-correlation function $C_{a b}\left(\nu ; t, t^{\prime}\right)$ from the time-resolved spectra, using the conventional definition of the intensity-intensity correlation 24]. The spectral cross-correlation was calculated for each spectrum at time $t$ with the spectrum at $t^{\prime}=-3 \mathrm{ps}$, as shown in Fig. 2(c), where spectra taken at 20 different locations on the sample were used to obtain an ensemble average.

For times directly after the arrival of the pump pulse, the time-correlation shows a reduction in amplitude, accompanied by a spectral shift, denoted hereafter by $\delta \nu_{0}$, of $10.2 \pm 1.0 \mathrm{~cm}^{-1}$. Cross sections of Fig. 2(c) are shown in Fig. 3(a) at -3 ps (black dots) and 1 ps (red diamonds). The spectral autocorrelation can be calculated using [24]

$$
C_{a b}\left(\Delta \nu, \tau_{a}\right) \simeq N\left[|\eta L|^{2} /|\sinh (\eta L)|^{2}\right],
$$

with $\eta=D^{-\frac{1}{2}}\left(\tau_{a}^{-1}+2 \pi i \Delta \nu\right)^{1 / 2}$, where $D$ is the diffusion constant of the light, and $\tau_{a}=\left(v_{E} \alpha\right)^{-1}$ the absorption time, $v_{E}=3 D / \ell$ being the energy velocity. The normalization constant $N$ is used to fit Eq. (2) to our data 


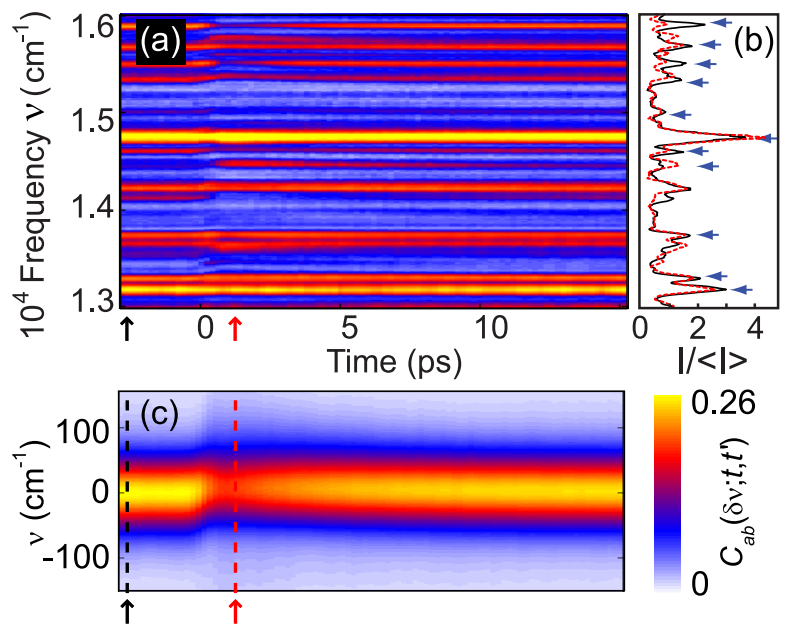

FIG. 2: (color online) (a) Map of time-resolved frequency speckle for sample 2 , with (b) spectra taken at -3 ps (black) and 1 ps (red) pump-probe time delay. Blue arrows indicate peaks selected for pseudomode analysis. (c) Map of experimental correlation $C_{a b}\left(\nu ; t, t^{\prime}\right)$.

given the reduced experimental speckle contrast. Equation (2) gives good agreement for the autocorrelation at -3 ps [line, black in Fig. 3(a)] for $D=15 \pm 2 \mathrm{~m}^{2} / \mathrm{s}$.

The reduction of the correlation between spectra at different times allows for an estimate of the dephasing time. As shown in Ref. 8, dephasing processes affect the average probability of light transport through an exponential decay $\exp \left(-t / \tau_{\gamma}\right)$ of the correlation between two amplitudes, where $\tau_{\gamma}$ is the dephasing time. The effects of dephasing and average refractive index change 25] can thus be introduced into the correlation function by replacing $\eta$ in Eq. (2) by the new

$$
\eta_{\gamma}=D^{-\frac{1}{2}}\left[\tau_{a}^{-1}+\tau_{\gamma}^{-1}+2 \pi i\left(\Delta \nu+\nu \frac{\Delta n_{e}}{n_{e}}\right)\right]^{1 / 2} .
$$

We note that the normalization factor $N$ only depends on the variance of the two individual configurations, which is not affected by the dephasing or the effective index change. We fitted the reduction in correlation amplitude by the dephasing model of Eq. (3). Good quantitative agreement is found [thick line, red in Fig. 3(a)] for a dephasing time $\tau_{\gamma}=1.2 \pm 0.2 \mathrm{ps}$ and a change in the average refractive index of the scattering medium of $\Delta n_{e} / n_{e}=(-5.8 \pm 2) \times 10^{-4}$.

We considered, next to the loss of correlation, also the effect of ultrafast excitation on individual pseudomodes. Pseudomodes are defined as the transmission modes of the system, which take on the form of spectral resonances, with a spectral width determined by the leakage of the state to free space. The spectrum of pseudomodes plays an important role in theories of localization [26, 27] and random lasers 28]. We have traced the time evolution of a subset of pseudomodes selected from
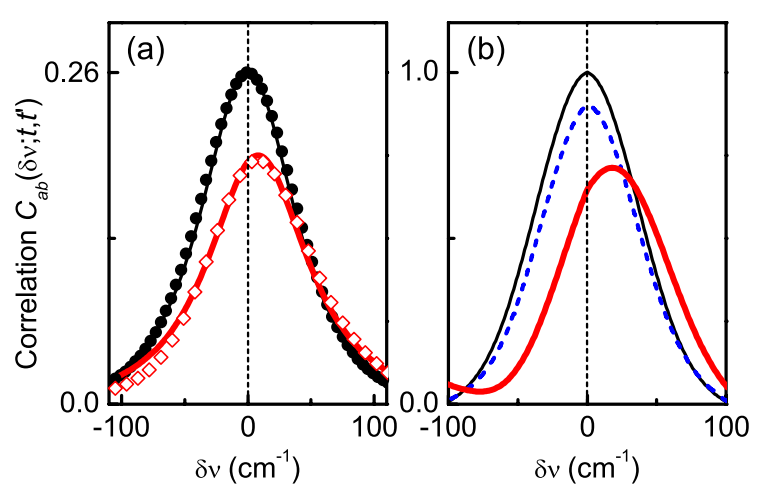

FIG. 3: (color online) (a) Cross sections of Fig. 2(c) at delay times of -3 ps (dots, black), and 1 ps (diamonds, red). Lines represent fits using Eq. (2), without (black) and with (red line) dephasing model with $\tau_{\gamma}=1.2 \pm 0.2 \mathrm{ps}$. (b) Finiteelement calculations [23] of autocorrelation (line, black) and cross-correlations for a refractive index change $\Delta n_{\mathrm{GaP}}=-2 \times$ $10^{-3}+10^{-3} i$ (dashed line, blue), and an additional $10^{-3}$ size increase of individual scatterers (thick line, red).

our experimental spectra using an algorithm 29], including modes such as those indicated by the blue arrows in Fig. 2(b). The position and amplitude of each pseudomode was tracked as function of the pump-probe delay time, yielding a set of well-defined intensity changes and spectral shifts shown in Fig. $4(a, b)$. Each time delay $t$ represents a histogram, such as is shown in the right-hand panels of Fig. $4(a, b)$ for $t=1$ ps. A wide distribution of spectral and intensity variations is found with both decreasing and increasing values, which extend beyond the variation of the time-correlation in Fig. 2(c). A fraction of modes in Fig. 4(b) shows a negative frequency shift, which cannot be explained by an average refractive index change but which indicates a more chaotic change of the pseudomode spectrum.

We have investigated possible dephasing processes using a numerical finite-element (COMSOL) model of light propagation in a two-dimensional slab consisting of a random arrangement of dielectric cylinders 23]. The frequency cross-correlation was calculated between two configurations corresponding to the situations before and after excitation. Consistent with earlier observations 25], no decorrelation was found for only a small refractive change of around $10^{-3}$. However, our model calculations identified two other possible dephasing mechanisms: absorption and particle deformation. While absorption [dashed line, green in Fig. B(b) for $\Delta n_{\mathrm{GaP}}=$ $\left.-2 \times 10^{-3}+10^{-3} i\right]$ does not change the phase of individual light paths, it does lead to a redistribution of phasor weights in the total speckle field by suppression of contributions from long paths. This interpretation is only partially correct in time-resolved experiments, where paths are separated in time and only the finite duration of the probe pulse leads to mixing of paths with different amounts of absorption. We point out that inhomoge- 

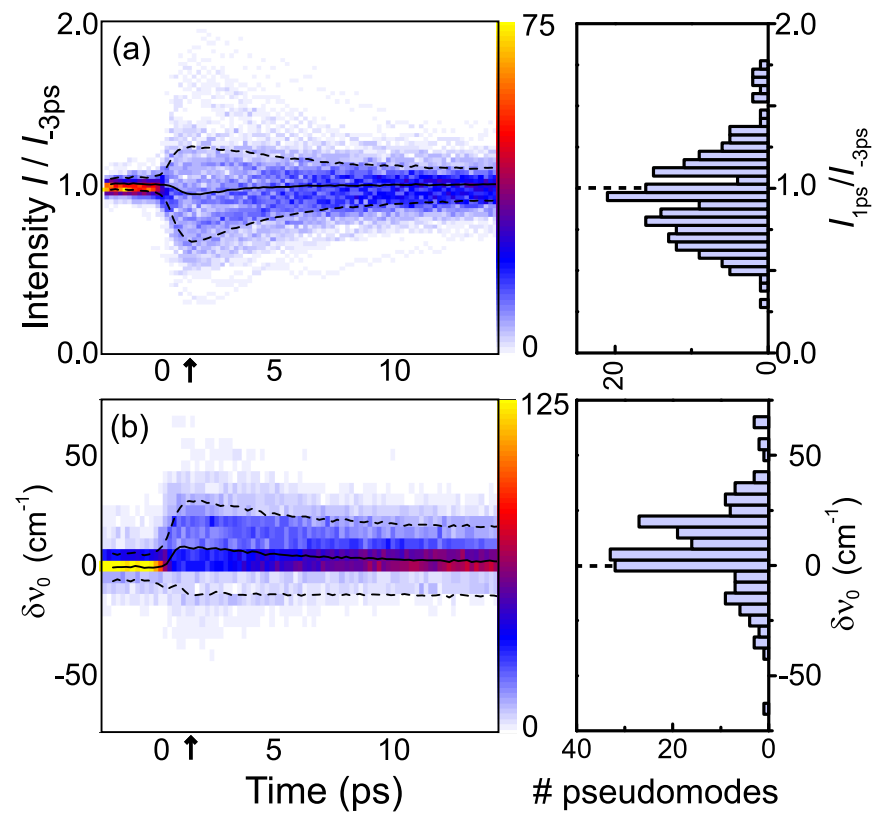

FIG. 4: (color online) Results of pseudomode analysis of 202 modes, yielding nonlinear intensity change $I / I_{-3 \mathrm{ps}}$ (a) and spectral shift $\delta \nu_{0}$ (b). (Red dashed lines) statistical average of the distributions. Arrows indicate time location of the histograms shown in right-hand panels. Lines indicate averages (solid) and standard deviations (dash) of the distributions.

neous absorption may also lead to variations between different paths of equal length and thus to dephasing.

We explored another possible dephasing mechanism caused by stress-induced deformations. It was found that a $10^{-3}$ increase of the scatterer size is sufficient to achieve quantitative agreement with experiment [thick line, red in Fig. [3(b)]. Such deformations are likely to occur under the typical conditions of high power pulsed laser excitation of solids [30], and may be enhanced by the particular morphology of the nanowires 31]. The effect of deformations is important as it represents the contribution of changes in the scattering matrix of individual scatterers. No dephasing was observed when randomly displacing each scatterer over $0.1 \mathrm{~nm}$.

Our analysis of pseudomode distributions and spectral correlations reveals that ultrafast dephasing enables reversible switching between random configurations involving thousands of scattering events. Although the intensity and frequency changes appear chaotic, they are highly reproducible over millions of pump-probe cycles. The observed pseudomode intensity modulations of up to $50 \%$ are large for the field of nanophotonics, and comparable to e.g. nonlinear switching of three-dimensional photonic crystals 32]. The similar decorrelation values for the two nanowire layers with different thicknesses 23 ] indicates that dephasing is limited by the pump absorption length, rather than by sample thickness; further optimization of dephasing using e.g. multiphoton excitation
[32], will be of importance for exploitation of these effects in applications. Deterministic control of pseudomodes may be combined with active gain media to produce novel types of ultrafast random lasers where the lasing modes can be manipulated using an external control pulse. The dephasing time of $\sim 1.2 \mathrm{ps}$ is of the order of the transport time through the layer, which paves the way for nonadiabatical control over light paths. Of particular interest here will be the possibility to break the phase coherence of correlated paths involved in mesoscopic transport, and ultimately of time-reversed light paths involved in photon localization.

We thank the ORC FASTlab and D. Kundys for support, and A. Lagendijk and S. Faez for fruitful discussions. This work was supported by EPSRC through grant $\mathrm{EP} / \mathrm{H} 019669 / 1$.

* Electronic address: O.Muskens@soton.ac.uk

[1] G. Lerosey et al., Science 315, 1120 (2007).

[2] I. M. Vellekoop et al., Nature Photon. 4, 320 (2010).

[3] A. Lagendijk, B. van Tiggelen, D. S. Wiersma, Physics Today 62, 24 (2009).

[4] H. Hu et al., Nature Phys. 4, 945 (2008).

[5] L. Sapienza et al., Science 327, 1352 (2010).

[6] T. Schwartz et al., Nature 446, 52 (2007).

[7] A.A. Golubentsev, Sov. Phys. JETP 59, 26 (1984).

[8] E. Akkermans, G. Montambeaux, Mesoscopic physics of electrons and photons (1st ed. Cambridge, 2007).

[9] F. A. Erbacher, R. Lenke, and G. Maret, Europhys. Lett. 21, 551 (1993).

[10] B. L. Altshuler, A. G. Aronov, D. E. Khmelnitsky, Solid State Comm. 39, 619 (1981).

[11] M. Kaveh, M. Rosenbluh, I. Freund, Nature 326, 778 (1987).

[12] D.J. Pine et al., Phys. Rev. Lett. 60, 1134 (1988).

[13] R. Yan, D. Gargas, and P. Yang, Nature Photon. 3, 569 (2009).

[14] B. Tian et al., Nature 449, 885 (2007).

[15] S. W. Boettcher et al., Science 327, 185 (2010).

[16] O. L. Muskens et al., Nano Lett. 9, 930 (2009).

[17] J. Gómez Rivas et al., Phys. Rev. E 63, 046613 (2001).

[18] F. Scheffold and G. Maret, Phys. Rev. Lett. 81, 5800 (1998).

[19] M. A. El-Sayed, Acc. Chem. Res. 37, 326 (2004).

[20] C. R. Carey et al., J. Phys. Chem. C, 113, 19077 (2009).

[21] O. L. Muskens et al., Nano Lett. 8, 2638 (2008).

[22] S. Rychnovsky et al., Opt. Lett. 19, 527 (1994).

[23] See supplementary material.

[24] R. Berkovits and S. Feng, Phys. Reports 238, 135 (1994).

[25] S. Faez, P. M. Johnson, A. Lagendijk, Phys. Rev. Lett. 103, 053903 (2009).

[26] D. J. Thouless, Phys. Rep., 13C, 93 (1974).

[27] S. E. Skipetrov, B. A. van Tiggelen, Phys. Rev. Lett. 96, 043902 (2006).

[28] Y. Ling et al., Phys. Rev. A 64, 063808 (2001).

[29] Modes were selected using an algorithm based on the condition that the normalized intensity shows a peak larger than 0.5 above its surroundings over a spectral range 
equal to two times the correlation width.

[30] C. Thomsen et al., Phys. Rev. B 34, 4129 (1986).

[31] S. O. Mariager et al., Nano Lett. 10, 2461 (2010).
[32] T.G. Euser et al., Phys. Rev. B 77, 115214 (2008). 